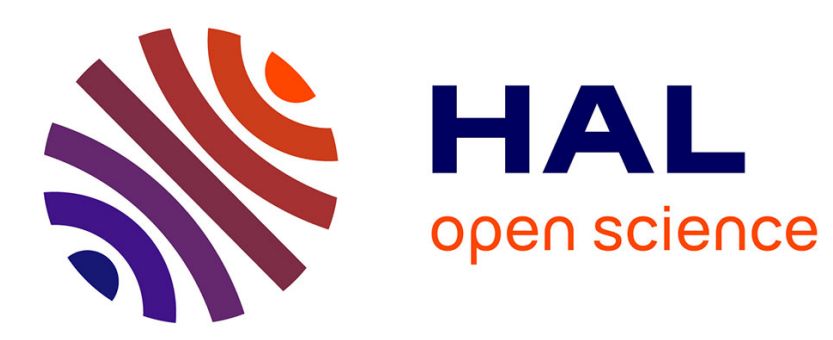

\title{
Pontryagin's principle in the control of semilinear elliptic variational inequalities
}

\author{
J. Frederic Bonnans, D. Tiba
}

\section{To cite this version:}

J. Frederic Bonnans, D. Tiba. Pontryagin's principle in the control of semilinear elliptic variational inequalities. [Research Report] RR-1134, INRIA. 1989. inria-00075425

\section{HAL Id: inria-00075425 \\ https://hal.inria.fr/inria-00075425}

Submitted on 24 May 2006

HAL is a multi-disciplinary open access archive for the deposit and dissemination of scientific research documents, whether they are published or not. The documents may come from teaching and research institutions in France or abroad, or from public or private research centers.
L'archive ouverte pluridisciplinaire HAL, est destinée au dépôt et à la diffusion de documents scientifiques de niveau recherche, publiés ou non, émanant des établissements d'enseignement et de recherche français ou étrangers, des laboratoires publics ou privés. 


\section{PRINCIPE DE PONTRYAGINE POUR LE CONTROLE D'INEQUATIONS} VARIATIONNELLES SEMILINEAIRES ELLIPTIQUES

\section{PONTRYAGIN'S PRINCIPLE IN THE CONTROL OF SEMILINEAR ELLIPTIC VARIATIONAL INEQUALITIES}

\section{J.F. BONNANS}

INRIA, Domaine de Voluceau

BP 105 - 78153 Rocquencourt - France
Dan TIBA

Department of Mathematics INCREST, Bd Pacii 220

79622 Bucuresti, Romania

Résumé Cet article traite des conditions nécessaires satisfaites par le contrôle optimal d'une inéquation variationnelle gourvernée par un opérateur semilinéaire de type elliptique et un opérateur maximal monotone $\beta$ dans $\mathbb{R} \times \mathbb{R}$. Une régularisation non classique de $\beta$ nous permet de formuler un problème perturbé pour lequel le contrôle originel est une $\varepsilon$-solution. Considérant les perturbations en aiguille et appliquant le principe d'Ekeland nous énonçons des conditions d'optimalité approchée sous forme de Pontryagine. Passant à la limite nous obtenons des conditions d'optimalité pour le problème de départ étendant celles obtenues pour les systèmes semilinéaires elliptiques et pour les inéquations variationnelles.

Abstract This paper deals with necessary conditions satisfied by the optimal control of a variational inequality governed by a semilinear operator of elliptic type and a maximal monotone operator $\beta$ in $\mathbb{R}$ $\times \mathbb{R}$. A non classical smoothing of $\beta$ allows us to formulate a perturbed problem for which the original control is an $\varepsilon$-solution. By considering the spike perturbations and applying Ekeland's principle we are able to state approximate optimality conditions in Pontryagin's form. Then passing to the limit we obtain some optimality conditions for the original problem extending those obtained for semilinear elliptic systems and for variational inequalities.

\section{TABLE}

I - Introduction

II - Setting of the problem

III - The smoothing process

IV - Study of the regularized problem

$\mathrm{V}$ - Returning to the original problem 


\section{I - INTRODUCTION}

In this paper we analyze the necessary conditions satisfied by optimal controls of a system governed by an operator which is the sum of a semilinear elliptic operator (the control entering in the nonlinear term) and of a maximal monotone operator; i.e. the state is solution of a semilinear variational inequality of elliptic type.

A large part of the litterature concerning the control of variational inequalities is devoted to the case when the maximal monotone operator involved in the state "equation" is actually the subdifferential of the indicatrix of a closed convex set; then the derivation of optimality conditions is close to the problem of derivation of projection onto a convex set and significant results have been obtained in this direction (see Mignot [14], Haraux [11]). However our study is more related to the studies concerned with the case when the monotone operator is only suppose to be local. We quote the pioneering work of Yvon [19] and the one of Saguez [15], in which regularization methods are used, i.e. a perturbed control problem is formulated with the help of a regularization of the maximal monotone operator, then optimality conditions are obtained for the solutions of the regularized problem, and finally passing to the limit one obtains some optimality conditions for the original problem. Such techniques have been widely extended in a series of paper of V. Barbu, synthetically exposed in his book [2], and we quote the work of Barbu and Tiba [3] for a recent development along this line. However the existence of optimal controls for the regularized problem imposes strong hypothesis on the data (see Ioffe and Tihomirov [12] about Pontryagin's principle in the control of ordinarydifferentialequations).

The novelty of this paper is twofold : we obtain the optimality conditions in Pontryagin's form, and our hypothesis are not far to be minimal. We essentially need the state equation to be well posed and differentiability of data with respect to the state. We restrict the study however to the case of an integral cost and only local constraints on the control.

Our results use those obtained recently by Casas and the first author [4], in which Pontryagin's principle is derived, but without the monotone term, and we use again regularization. However our hypotheses do not imply the existence of a solution of the perturbed problem. Rather we use Ekeland's principle, and for this purpose we have to prove that the original solution is an $\varepsilon$-solution of the perturbed problem.

This leads us to devise a new kind of approximation, extending the one in Kinderlehrer and Stampacchia [13], that we call $\varepsilon$-uniform approximation for the maximal monotone operators in $\mathbf{R} \times$ $\mathbb{R}$. Then the following striking property holds. If $\mathrm{u}$ is a control, $\mathrm{y}_{\mathrm{u}}$ and $\mathrm{y}_{\mathrm{u}}^{\varepsilon}$ the solutions of the original and perturbed state equation, then $\left\|_{y_{u}}-y_{u}\right\|_{\|_{\infty}} \leqslant \varepsilon$. As we give a constructive means to obtain smooth 
$\varepsilon$-uniform approximations, we suspect that this property might be useful in numerical computations. However in this paper uniform approximations are just used to obtain the stability of the optimal cost.

The paper is organized as follows. In part 2 we set the problem, state the main hypotheses and prove the well posedness of the state equations. Part 3 is devoted to $\varepsilon$ - uniform approximations.

Optimality conditions related to the perturbed problem are obtained in part 4 using Ekeland's principle. Then in part 5 we come back to the original problem and analyse the special case when the monotone operator is piecewise constant. The appendix contains the proof of the $\mathrm{W}^{2, \mathrm{~s}}(\Omega)$ regularity of the solution of a variational inequality.

\section{II - SETTING OF THE PROBLEM}

Let $\Omega$ be a bounded open set of $\mathbb{R}^{\mathbf{n}}$ with Lipschitz boundary $\Gamma$. We consider the following control system

$$
\left\{\begin{array}{l}
A y+\varphi(x, y(x), u(x))+\beta(y(x)) \ni 0 \quad \text { a.e. } x \text { in } \Omega \\
y=0 \text { on } \Gamma
\end{array}\right.
$$

Here $A$ is a differential operator of the form

$$
A y=-\sum_{i, j=1}^{n} \partial_{x_{i}}\left(a_{i j}(x) \partial_{x_{j}}(y(x))\right),
$$

associated to the positive bilinear form : $\mathrm{H}_{0}^{1}(\Omega) \times \mathrm{H}_{0}^{1}(\Omega) \rightarrow \mathbb{R}$

$$
a(y, z)=\sum_{i, j=1}^{n} a_{i j}(x) \frac{\partial y}{\partial x_{j}}(x) \frac{\partial z}{\partial x_{i}}(x) d x,
$$

the control $u$ is a.e. in $K \subset \mathbb{R}, \varphi$ is a mapping $: \Omega \times \mathbb{R} \times K \rightarrow \mathbb{R}$, and $\beta$ is a maximal monotone graph in $\mathbb{R} \times \mathbb{R}$ (see Brezis [7], Barbu [2]) such that dom $(\beta) \ni 0$. The solution y of (2.1) is called the state. Let $\mathrm{L}$ be a mapping $: \Omega \times \mathbb{R} \times \mathrm{K} \rightarrow \mathbb{R}$. The criterion that we will consider is

$$
J(y, u)=\int_{\Omega} L(x, y(x), u(x)) d x .
$$

Hence the control problem is

$$
\min J(y, u) \text { s.t. }(2.1) \text { and } u(x) \in K \text {, a.e. on } \Omega \text { : }
$$


We now make some assumptions in order to give a precise meaning to problem (2.3). Here for $i$ $\in \mathbb{N}, C_{i}$ are strictly positive constants and $\eta_{i}$ are nondecreasing mappings $: \mathbf{R}^{+} \rightarrow \mathbf{R}^{+}$. Also by $M_{i}($.) we will denote given elements of $L s(\Omega)$, where $s \geqslant 2$ satisfies also $s>n / 2$ (hence $s=2$ is convenient if $n \leqslant 3$ ). We assume the following :

$$
\begin{aligned}
& a_{i j}(.) \quad i, j=1, n \text { are continuously differentiable on } \bar{\Omega}, \\
& \sum_{i, j=1}^{n} a_{i j}(x) \xi_{i} \xi_{j} \geqslant C_{1} \sum_{i=1}^{n}\left(\xi_{i}\right)^{2}, \forall x \in \Omega, \\
& |\varphi(x, 0, u)| \leqslant M_{1}(x)+C_{2}|u|, \\
& 0 \leqslant \varphi_{y}^{\prime}(x, y, u) \leqslant\left[M_{2}(x)+C_{3}|u|\right] \eta_{1}(|y|), \\
& I L(x, 0, u)\left|\leqslant M_{3}(x)+C_{4}\right| u \mid, \\
& \mathbb{L}{ }_{y}^{\prime}(x, y, u) \mid \leqslant\left[M_{4}(x)+C_{5}|u|\right] \eta_{2}(|y|) .
\end{aligned}
$$

We will say that $u \in L^{S}(\Omega)$ is feasible (for problem (2.3)) whenever $u(x) \in K$ a.e. and the mapping $(x, y) \rightarrow(\varphi(x, y, u(x)), L(x, y, u(x)))$ satisfies the conditions of Caratheodory, i.e. is continuous with respect to $y$, a.e. $x \in \Omega$, and is measurable as a function of $x$ for all $y$. These conditions imply that the mapping $x \rightarrow(\varphi(x, y(x), u(x)), L(x, y(x), u(x))$ is measurable when $x \rightarrow y(x)$ is itself measurable. Let us define $\mathrm{Y}=\mathrm{W}^{2, \mathrm{~s}(\Omega)} \cap \mathrm{H}_{0}^{1}(\Omega)$. By Sobolev's imbedding Theorem W2,s $(\Omega)$ is compactly imbedded in $C^{\tau}(\bar{\Omega})$ (the space of Hölderian mappings with modulus $\tau$ ) with $\tau$ $=2-\mathrm{n} / \mathrm{s}>0$.

Theorem 2.1 There exists $\mathrm{C}_{6}>0$ such that for any feasible control $\mathrm{u}$, equation (2.1) has a unique solution $\mathrm{y}=\mathrm{y}_{\mathrm{u}}$ in $\mathrm{Y}$ and $\|y\|_{Y} \leqslant \mathrm{C}_{6}\left(1+\|\mathrm{u}\|_{\mathrm{L}} \mathrm{s}(\Omega) \cdot \mathrm{a}\right.$

Proof We may assume that $\beta(0) \ni 0$. First assume that $\operatorname{dom}(\beta)=\mathbb{R}$ and that $\beta$ is Lipschitz and continuously differentiable. Changing $\varphi(., y, u)+\beta(y)$ into $\varphi(., y, u)$ we may apply the result of [4]. We deduce that (2.1) has a (unique) solution in $\mathrm{H}_{0}^{1}(\Omega) \cap \mathrm{L}^{\infty}(\Omega)$. Let us define $\hat{\mathrm{y}}(\mathrm{x}), \tilde{\mathrm{y}}(\mathbf{x})$ as mappings given by the mean value Theorem :

$$
\begin{aligned}
& \varphi_{y}^{\prime}(., \hat{y}(x), u(x)) y(x)=\varphi(., y(x), u(x))-\varphi(., 0, u(x)), \\
& \beta_{y}^{\prime}(\tilde{y}(x)) y(x)=\beta(y(x))-\beta(0)=\beta(y(x)),
\end{aligned}
$$

and we have $\max (\hat{\mid y}(x)|,| \tilde{y}(x) \mid) \leqslant|y(x)|$ a.e. . Define 


$$
\psi(x)=\varphi_{y}^{\prime}(., \hat{y}(x), u(x))+\beta_{y}^{\prime}(\tilde{y}(x))
$$

Then $y(x)$ satisfies the following linear system :

$$
\left\{\begin{array}{l}
A y+\psi(x) y=-\varphi(., 0, u) \text { in } \Omega \\
y=0 \text { on } \Gamma
\end{array}\right.
$$

From $y \in L^{\infty}(\Omega)$ (hence $\hat{y}$ and $\tilde{y}$ bounded) and (2.7) we deduce that $\psi(x) \in L^{s}(\Omega)$. This and (2.6) allow to apply Lemma 3.2 of [4] ; we deduce a bound of $y$ in $L^{\infty}(\Omega)$ independent of $\beta$. We deduce with (2.6) (2.7) a bound of $A y+\beta(y)=-\varphi(., y, u)$ in $L^{s}(\Omega)$, hence a bound of $y$ in $W^{2}, s(\Omega)$ (by the results of the Appendix) independent on $\beta$.

When $\boldsymbol{\beta}$ is a general maximal monotone graph in $\mathbb{R} \times \mathbb{R}$ it is a standard trick to approximate it (via Yosida's approximation and convolution with a smoothing kernel : see [2]) with a Lipschitz $\mathrm{C}^{1}$ monotone function. Passing to the limit then is a now well known process (see again [2]).

Remark 2.1 In [4] $a_{\mathrm{ij}}$ is only assumed to be in $L^{\infty}(\Omega)$ (instead of (2.4)). Hypothesis (2.4) allows us to obtain the additional $\mathrm{W}^{2}, \mathrm{~s}(\Omega)$ regularity.

\section{III - THE SMOOTHING PROCESS}

\section{III.1 The approximate operator $\beta_{\varepsilon}$}

We present a way of computing approximations of $\beta$ that has the properties stated in the introduction. First we state the conditions that should be satisfied by the approximation, and prove that under these conditions the approximate state is well defined and is close in the $\mathrm{L}^{\infty}$ norm to the solution of the original variational inequality. Then we give a constructive way to obtain a $\mathrm{Cl}^{\mathbf{l}}$ approximation that satisfies these conditions.

We will say that for $\varepsilon>0$, a maximal monotone in $\mathbb{R} \times \mathbb{R}$ graph $\beta_{\varepsilon}$ is an $\varepsilon$-uniform approximation to $\beta$ if $\beta_{\varepsilon}$ satisfies the following two conditions :

$$
\text { i) } \beta(y+\varepsilon) \geqslant \beta_{\varepsilon}(y) \geqslant \beta(y-\varepsilon), \forall y \in \mathbb{R} \text {, }
$$

$$
\text { ii) } \operatorname{dom}\left(\beta_{\varepsilon}\right) \supset \operatorname{dom}(\beta) \text {. }
$$

Here in (3.1i) we view $\beta$ and $\beta_{\varepsilon}$ as multivalued operators extended on $\mathbb{R}$ with value $-\infty$ on the left of their domain and $+\infty$ on the right of their domain, and the inequality for sets means 
$\xi \geqslant \eta \geqslant v, \forall \xi \in \beta(y+\varepsilon), \eta \in \beta_{\varepsilon}(y), v \in \beta(y-\varepsilon)$.

To an $\varepsilon$-uniform approximation $\beta_{\varepsilon}$ we associate the perturbed equation

$$
\left\{\begin{array}{l}
A y_{\varepsilon}+\varphi\left(x, y_{\varepsilon}(x), u(x)\right)+\beta_{\varepsilon}\left(y_{\varepsilon}\right) \ni 0 \text { in } \Omega \\
y_{\varepsilon}=0 \text { on } \Gamma
\end{array}\right.
$$

Theorem 3.1 Let $\mathrm{u}$ be feasible for problem (2.3). Then (3.2) has a unique solution $\mathrm{y}_{\varepsilon}$ in $\mathrm{Y}$, and $\| \mathrm{y}_{\varepsilon}$ $-y \|_{\infty} \leqslant \varepsilon .0$

Proof. That (3.2) has a unique solution $y_{\varepsilon}$ in $Y$ is a consequence of (3.1ii) and Theorem 2. We now prove that $\mathrm{y}_{\varepsilon}(\mathrm{x}) \leqslant \mathrm{y}(\mathrm{x})+\varepsilon$ on $\Omega$ (that $\mathrm{y}(\mathrm{x}) \leqslant \mathrm{y}_{\varepsilon}(\mathrm{x})+\varepsilon$ being proved in the same way). Define $\mu_{\varepsilon}(y+\varepsilon)$ as a measurable selection of $\beta_{\varepsilon}(y+\varepsilon)$. We define (we drop the variable $x \in \Omega$ )

$$
\begin{aligned}
& \mathrm{z}:=\min \left(0, \mathrm{y}+\varepsilon-\mathrm{y}_{\varepsilon}\right), \\
& \delta:=\mathrm{a}(\mathrm{y}, \mathrm{z})+\oint_{\Omega} \varphi(., \mathrm{y}+\varepsilon, \mathrm{u}) \mathrm{zdx}+\oint_{\Omega} \mu_{\varepsilon}(\mathrm{y}+\varepsilon) \mathrm{zdx} .
\end{aligned}
$$

Indeed $\mathrm{z}$ is in $\mathrm{H}_{0}^{1}(\Omega)$ hence the two first integrals of $\delta$ are well defined. let us prove that this is the case for the third one, too. To the product $\mu_{\varepsilon}(y(x)+\varepsilon) z(x)$ we give the value 0 if $z(x)=0$. Otherwise $y(x)<y(x)+\varepsilon<y_{\varepsilon}(x)$ hence with (3.1i) and using the monotonicity of $\beta_{\varepsilon}$ :

$$
\beta(y(x)) \leqslant \beta_{\varepsilon}(y(x)+\varepsilon) \leqslant \beta_{\varepsilon}\left(y_{\varepsilon}(x)\right),
$$

and consequently, a.e. on $\Omega$, defining

$$
\begin{aligned}
& \mu(y(x))=-(A y+\varphi(., y, u)), \\
& \mu_{\varepsilon}\left(y_{\varepsilon}(x)\right)=-\left(A y_{\varepsilon}+\varphi\left(., y_{\varepsilon}, u\right)\right)
\end{aligned}
$$

we have using (3.3)

$$
\left|\mu_{\varepsilon}(y(x)+\varepsilon) z(x)\right| \leqslant\left(|\mu(y(x))|+\left|\mu_{\varepsilon}\left(y_{\varepsilon}(x)\right)\right|\right)|z(x)|
$$

The right hand side being in $\mathrm{L}^{1}(\Omega)$ and $\mathrm{z}$ being in $\mathrm{L}^{\infty}, \delta$ is well defined. Extracting from $\delta$ the original state equation we get 


$$
\begin{aligned}
\delta & =\oint_{\Omega}^{[A y+\varphi(x, y, u)+\mu(y)] z d x} \\
& +\oint_{\Omega}^{[\varphi(x, y+\varepsilon, u)-\varphi(x, y, u)] z d x} \\
& +\oint_{\Omega}^{\left[\mu_{\varepsilon}(y+\varepsilon)-\mu(y)\right] z d x} .
\end{aligned}
$$

The first integral is null and the two others are nonpositive (by the negativity of $z,(2.7)$ and (3.3)), hence $\delta \leqslant 0$. Subtracting now the integral of the product of the perturbed state equation by $z$ from $\delta$ (and noticing that $\nabla(y+\varepsilon)=\nabla y$ ) we get

$$
\begin{aligned}
\delta & =a\left(y+\varepsilon-y_{\varepsilon}, z\right)+\left\{\left[\varphi(., y+\varepsilon, u)-\varphi\left(., y_{\varepsilon}, u\right)\right] z d x\right. \\
& +\oint_{\Omega}\left[\mu_{\varepsilon}(y+\varepsilon)-\mu_{\varepsilon}\left(y_{\varepsilon}\right)\right] z d x .
\end{aligned}
$$

By the definition of $z$ and $(2.7),\left(\varphi(., y+\varepsilon, u)-\varphi\left(., y_{\varepsilon}, u\right)\right) z$ and $\left(\mu_{\varepsilon}(y+\varepsilon)-\mu_{\varepsilon}\left(y_{\varepsilon}\right) z_{\varepsilon}\right.$ are nonnegative a.e., hence

$$
0 \geqslant a\left(y+\varepsilon-y_{\varepsilon}, z\right)=a(z, z)
$$

As $z=0$ on $\Gamma$, we deduce that $z=0$ on $\Omega$, hence $y_{\varepsilon} \leqslant y+\varepsilon$ a.e., as was to be proved.

\section{III.2. Construction of $\beta_{\varepsilon}$}

The set of operators satisfying (3.1) is not empty as it contains $\beta$ itself. However we want to obtain a $C^{1}$ approximation $\beta_{\varepsilon}$ in order to obtain a first order optimality system on the perturbed system. For this purpose we consider a regularizing kernel, i.e. a $\mathrm{C}^{\infty}$ function $\rho: \mathbf{R} \rightarrow \mathbf{R}$ with support in $[0,1]$ satisfying $\rho \geqslant 0$ and $\int_{0}^{1} \rho(s) \mathrm{d} s=1$. We construct approximations in five basic cases, then explain how to deal with the general case.

Case $1 \operatorname{Dom}(\beta)=\mathbb{R}$. In this case we simply consider

$$
\beta_{\varepsilon}(s)=\int_{0}^{1} \beta(s+\varepsilon \sigma) \rho(\sigma) d \sigma .
$$

Obviously $\beta_{\varepsilon}$ has all desired properties. 
Case $2 \beta(s)=0$ on $\operatorname{dom}(\beta)=\left(-\infty, s_{0}\right]$. We take

$$
\beta_{\varepsilon}(s)=\left\{\begin{array}{l}
0 \text { if } s<s_{0}, \\
\operatorname{tg} \frac{\pi}{2 \varepsilon}\left(s-s_{0}\right) \text { if } s \in\left[s_{0}, s_{0}+\varepsilon\right) \\
\varnothing \text { if } s \geqslant s_{0}+\varepsilon
\end{array}\right.
$$

Then as $\beta_{\varepsilon}$ is increasing and $\beta_{\varepsilon}(s) \nearrow+\infty$ when $s \nearrow s_{0}+\varepsilon, \beta_{\varepsilon}$ is maximal monotone. Obviously $\beta_{\varepsilon}$ is $C^{1}$ and satisfies (3.1).

Case $3 \beta(0)=0$ on dom $(\beta)=\left[\mathrm{s}_{0},+\infty\right)$. Similarly to case 2 we take

$$
\beta_{\varepsilon}(s)=\left\{\begin{array}{l}
\varnothing \text { if } s \leqslant s_{0}-\varepsilon, \\
\operatorname{tg} \frac{\pi}{2 \varepsilon}\left(s-s_{0}\right) \text { if } s \in\left(s_{0}-\varepsilon, s_{0}\right], \\
0 \text { if } s \geqslant s_{0} .
\end{array}\right.
$$

Case $4 \operatorname{dom}(\beta)=\left(-\infty, s_{0}\right)$. Necessarily $\beta(s) \not+\infty$ as $s>s_{0}$ otherwise $\beta$ would not be maximal monotone. Then we approximate $\beta$ as follows

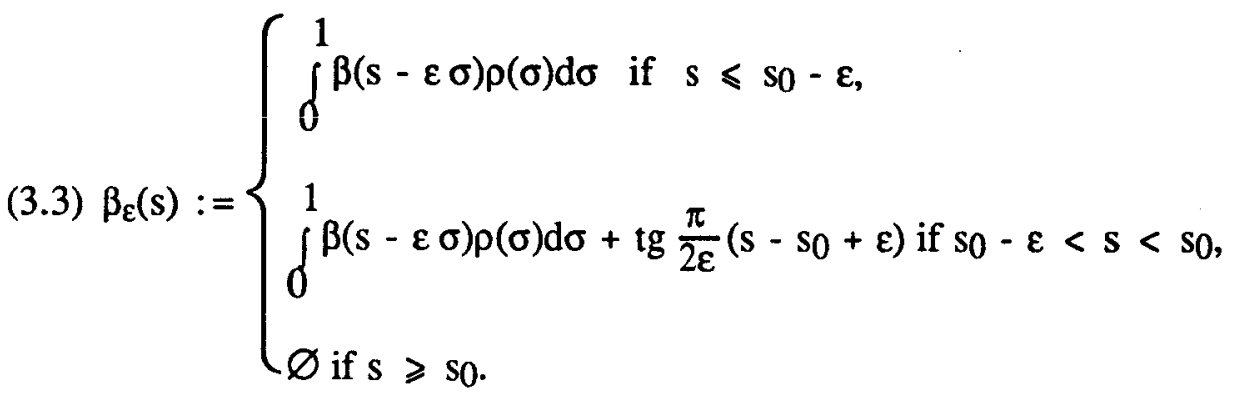

This is a well defined $C^{1}$ and monotone function in $\left(-\infty, s_{0}\right)$ and $\beta_{\varepsilon}(s)>\infty$ when $s>s_{0}$. Hence $\beta_{\varepsilon}$ is maximal monotone, and (3.1) is satisfied.

Case $5 \operatorname{dom}(\beta)=\left(\mathrm{s}_{0},+\infty\right)$. Similarly to case 4 we define

$$
\beta_{\varepsilon}(s):=\left\{\begin{array}{l}
\varnothing \text { if } s \leqslant s_{0}, \\
1 \\
\left\{\beta(s+\varepsilon \sigma) \rho(\sigma) d \sigma+\operatorname{tg} \frac{\pi}{2 \varepsilon}\left(s-s_{0}-\varepsilon\right) \text { if } s_{0}<s \leqslant s_{0}+\varepsilon,\right. \\
1 \\
\left\{\beta(s+\varepsilon \sigma) \rho(\sigma) d \sigma \text { if } s_{0}+\varepsilon<s .\right.
\end{array}\right.
$$

In order to deal with the general case we can take advantage of the easy-to-prove following property : 


$$
\left\{\begin{array}{l}
\text { If } \beta^{1} \text { and } \beta^{2} \text { are maximal monotone graphs in } \mathbb{R} \times \mathbb{R} \text { as well as their sum } \\
\beta:=\beta^{1}+\beta^{2} \text {, and } \beta_{\mathcal{E}}^{1}, \beta_{\varepsilon}^{2} \text { are a uniform approximation of } \beta^{1}, \beta^{2} \text { then } \beta_{\varepsilon}^{1}+\beta_{\varepsilon}^{2} \\
\text { is a uniform approximation of } \beta .
\end{array}\right.
$$

Now if $\beta$ is any maximal monotone graphs in $\mathbb{R} \times \mathbb{R}$, taking $s_{1}$ in the interior of $\operatorname{dom}(\beta)$ with $\beta\left(s_{1}\right)$ single-valued (we exclude the trivial case $\operatorname{dom}(\beta)=\{0\}$ ) it is easy to decompose $\beta$ as $\beta^{1}+\beta^{2}$ with $\beta^{1}, \beta^{2}$ maximal monotone and $\operatorname{dom}\left(\beta_{1}\right) \supset\left(-\infty, s_{1}\right], \operatorname{dom}\left(\beta_{2}\right) \supset\left[s_{1}, \infty\right)$. If $\operatorname{dom}\left(\beta^{1}\right)$ is of the form $\left(-\infty, s_{0}\right)$ we saw in case 4 how to approximate $\beta^{1}$. If $\operatorname{dom}\left(\beta^{1}\right)$ is of the form $\left(-\infty, s_{0}\right]$ then put

$$
\beta^{1, a}(s)=\left\{\begin{array}{l}
\beta^{1}(s) \text { if } s<s_{0}, \\
\lim _{s>s_{0}} \beta^{1}(s) \text { if } s \geqslant s_{0},
\end{array}\right.
$$

and

$$
\beta 1, b(s)=\left\{\begin{array}{l}
0 \text { if } s<s, \\
{[0, \infty) \text { if } s=s_{0},} \\
\varnothing \text { if } s>s_{0}
\end{array}\right.
$$

Then $\beta^{1}=\beta^{1, a}+\beta^{1, b}$; the approximation of $\beta^{1, a}$ and $\beta^{1, b}$ is discussed in cases 1 and 2 . Using the decomposition property 3.6 we obtain the desired approximation of $\beta^{1}$, and similarly for $\beta^{2}$.

\section{IV - STUDY OF THE REGULARIZED PROBLEM}

\section{IV.1 Stability of the infimal cost}

We consider the optimization problem when $\beta$ is approximated by a smooth $\varepsilon$-uniform approximation $\beta_{\varepsilon}$ :

$$
\min \mathrm{J}(\mathrm{y}, \mathrm{u}) \text { s.t. (3.2) and } \mathrm{u}(\mathrm{x}) \in \mathrm{K} \text {, a.e. on } \Omega \text {. }
$$

Assuming that $\mathrm{K}$ is bounded, we will prove that any solution of the original problem (2.3) is a suboptimal solution of the perturbed problem (4.1). Then using Ekeland's principle we will obtain some necessary optimality conditions.

Theorem 4.1 When $\varepsilon>0$ then the following holds : 
(i) For any feasible control $u$, the solution $y_{u}^{\varepsilon}$ of the perturbed state equation (2.3) is well defined and

$$
\mathrm{J}\left(\mathrm{y}_{\mathrm{u}}^{\varepsilon}, \mathrm{u}\right)=\mathrm{J}\left(\mathrm{y}_{\mathrm{u}}, \mathrm{u}\right)+0(\varepsilon)
$$

with $10(\varepsilon) \mid \leqslant C_{7} \varepsilon$, and we may take $C_{7}$ independent of $u$ if $\mathrm{K}$ is bounded.

(ii) If $\mathrm{K}$ is bounded then $|\inf (4.1)-\inf (2.3)| \leqslant C_{7} \varepsilon$.

Proof Let $\mathrm{u}$ be a feasible control. That $\mathrm{y}_{\mathrm{u}}^{\mathcal{E}}$ is well defined has already being proved in section 3 , and we know that $\left\|y_{u}^{\varepsilon}-y_{u}\right\|_{\infty} \leqslant \varepsilon$. Using (2.9) we deduce that for $\varepsilon \leqslant 1$

$$
\left.\mathrm{J}\left(\mathrm{y}_{\mathrm{u}}^{\varepsilon}, \mathrm{u}\right)-\mathrm{J}\left(\mathrm{y}_{\mathrm{u}}, \mathrm{u}\right) \mid \leqslant \varepsilon \varepsilon_{\Omega}\left[\mathrm{M}_{4}(\mathrm{x})+\mathrm{C}_{5} \mid \mathrm{l}(\mathrm{x})\right]\right] \eta_{2}\left(\left\|\mathrm{y}_{\mathrm{u}}\right\|_{\infty}+1\right) d x
$$

i.e. $\left|J\left(y_{u}^{\varepsilon}, u\right)-J\left(y_{u}, u\right)\right| \leqslant C_{7} \varepsilon$ for some $C_{7}>0$. Also if $K$ is bounded, then $\| u l_{\infty}$ and $\left\|y_{u}\right\|_{\infty}$ are also bounded. In this case, we may suppose that $C_{7}$ does not depend on $u$. This proves (i). Taking a sequence $\mathrm{u}^{\mathrm{k}}$ such that $\left.\mathrm{J}\left(\mathrm{y}^{\mathrm{k}}, \mathrm{u}^{\mathrm{k}}\right)\right) \rightarrow \inf (2.3)\left(\right.$ resp. $\mathrm{J}\left(\mathrm{y}_{\mathrm{u}^{\ell}}^{\varepsilon}, \mathrm{u}^{\ell}\right) \rightarrow \inf (4.1)$ ) we obtain inf (4.1) $\leqslant$ $\inf (2.3)+C_{7} \varepsilon\left(\right.$ resp. $\left.\geqslant \inf (2.3)-C_{7 \varepsilon}\right)$. This proves (ii).

For any $\alpha \geqslant 0$ we will say that a feasible control $u$ is an $\alpha$-solution of (2.3) (resp. (4.1)) if $\mathrm{J}\left(\mathrm{y}_{\mathrm{u}}, \mathrm{u}\right) \leqslant \inf (2.3)+\alpha\left(\operatorname{resp} .\left(\mathrm{J}\left(\mathrm{y}_{\mathrm{u}}^{\varepsilon}, \mathrm{u}\right) \leqslant \inf (4.1)+\alpha\right)\right.$. Then statement (ii) of Theorem 4.2 implies the following : for any $\alpha \geqslant 0$, any $\alpha$-solution of (2.3) is a $\left(\alpha+C_{7} \varepsilon\right)$-solution of (4.1).

IV.2 Approximate optimality conditions

We first recall Ekeland's principle, then use it on the regularized problem in order to derive some optimality conditions (depending on $\varepsilon$ ) for $\alpha$-solutions of the original problem.

Theorem 4.2 (Ekeland's principle : see [9], [10]). Let (E,d) be a complete metric space and F a l.s.c. mapping : $E \rightarrow \mathbb{R} \cup\{+\infty\}$ and for $\varepsilon>0$ given, let $\mathrm{e}^{\varepsilon} \in \mathrm{E}$ be such that $\mathrm{F}\left(\mathrm{e}^{\varepsilon}\right) \leqslant \inf \mathrm{F}+\varepsilon^{2}$. Then there exists $\mathrm{e}^{\prime} \in \mathrm{E}$ satisfying

$$
\begin{aligned}
& \mathrm{F}\left(\mathrm{e}^{\prime}\right) \leqslant \mathrm{F}\left(\mathrm{e}^{\varepsilon}\right) \\
& \mathrm{d}\left(\mathrm{e}^{\varepsilon}, \mathrm{e}^{\prime}\right) \leqslant \varepsilon \\
& \mathrm{F}\left(\mathrm{e}^{\prime}\right) \leqslant \mathrm{F}(\mathrm{e})+\varepsilon \mathrm{d}\left(\mathrm{e}, \mathrm{e}^{\prime}\right), \forall \mathrm{e} \in \mathrm{E} . \square
\end{aligned}
$$


We define the Hamiltonian

$$
H(x, y, u, p)=L(x, y, u)-p \varphi(x, y, u)
$$

We will use Theorem 4.2 with the space and the metric

$$
\begin{aligned}
& E=\left\{u \in L^{\infty}(\Omega) ; u(x) \in K \text {, a.e. } x \in \Omega\right\} \\
& d(u, v)=\operatorname{mes}\{x \in \Omega ; u(x) \neq v(x)\} .
\end{aligned}
$$

That $\mathrm{d}$ is actually a metric is well known (see [10]). We define the costate associated to a control $\mathrm{u}$ for problem (4.1) as the solution of

$$
\left\{\begin{array}{l}
A^{*} p^{\varepsilon}+\varphi_{y}^{\prime}\left(., y_{u^{\varepsilon}}^{\varepsilon}, u\right) p^{\varepsilon}+\beta_{\varepsilon}^{\prime}\left(y_{u^{\varepsilon}}^{\varepsilon} p^{\varepsilon}=L_{y}^{\prime}\left(., y_{u^{\prime}}^{\varepsilon}, u\right) \text { in } \Omega\right. \\
p^{\varepsilon}=0 \text { on } \Gamma
\end{array}\right.
$$

where $A^{*}$ is the formal adjoint operator of $A$. This linear equation has a unique solution in $\mathrm{Y}$ (this is a consequence of Theorem 2.1, (2.7), (2.9) and $y \in Y$ ).

Theorem 4.3 We assume that $\mathrm{K}$ is bounded. Let $\mathrm{u}$ be an $\alpha$-solution of (2.3). Put $\alpha^{\varepsilon}:=\alpha+\mathrm{C}_{7} \varepsilon$ where $C_{7}$ is given by Thm 4.1. Then for each $\varepsilon>0$ there exists $u^{\varepsilon}, \alpha^{\varepsilon}$-solution of (4.1), satisfying $\mathrm{d}\left(\mathrm{u}, \mathrm{u}^{\varepsilon}\right) \leqslant\left(\alpha^{\varepsilon}\right)^{1 / 2}$ and such that $\forall \mathrm{v} \in \mathrm{K}$, denoting by $\mathrm{y}^{\varepsilon}, \mathrm{p}^{\varepsilon}$ the state and costate associated to $\mathrm{u}^{\varepsilon}$ for problem (4.1), the following relation is satisfied :

$$
H\left(., y^{\varepsilon}, u, p^{\varepsilon}\right) \leqslant H\left(., y^{\varepsilon}, v, p^{\varepsilon}\right)+\left(\alpha^{\varepsilon}\right)^{1 / 2} \text {, a.e. on } \Omega . \square
$$

Proof By Theorem 4.1, $\mathrm{u}$ is an $\alpha^{\varepsilon}$-solution of (4.1). We prove that the mappings $\mathrm{u} \rightarrow \mathrm{y}_{\mathrm{u}^{\prime}}^{\varepsilon}$ $\mathrm{u} \rightarrow \mathrm{J}\left(\mathrm{y}_{\mathrm{u}}^{\varepsilon}, \mathrm{u}\right)$ (here $\mathrm{y}_{\mathrm{u}}^{\varepsilon}$ is the solution of the perturbed state equation (3.2)) are continuous $(\mathrm{E}, \mathrm{d}) \rightarrow \mathrm{Y}$ weak and $(E, d) \rightarrow \mathbb{R}$, respectively. If $\left\{u^{k}\right\}$ is a sequence of feasible controls and $d\left(u^{k}, u\right) \rightarrow 0$, denoting by $\mathrm{y}^{\mathrm{k}}$ the solution of (3.2), we have that $\left\{\mathrm{y}^{\mathrm{k}}\right\}$ is bounded in $\mathrm{Y}$. For some subsequence again denoted $\left\{y^{k}\right\}$ one has $y^{k} \rightarrow y$ in $Y$ for some $y$ in $Y$. Hence $y^{k} \rightarrow y$ in $L^{\infty}(\Omega)$. With (2.6), (2.7) and Lebesgue's Theorem we deduce that $\varphi\left(., y^{k}, u^{k}\right) \rightarrow \varphi\left(., y^{k}, u^{k}\right)$ in $L^{s}(\Omega)$ and $\beta_{\varepsilon}\left(y^{k}\right) \rightarrow \beta_{\varepsilon}(y)$ in $L^{\infty}(\Omega)$. Passing to the limit in (3.2) we deduce that all the sequence $\left\{y^{k}\right\}$ weakly converges in $Y$ towards $y_{u^{\prime}}^{\varepsilon}$, i.e. $\mathrm{u} \rightarrow \mathrm{y}_{\mathrm{u}}^{\varepsilon}$ is continuous $(\mathrm{E}, \mathrm{d}) \rightarrow \mathrm{Y}$ weak. With $(2.8),(2.9)$ and Lebesgue's Theorem we deduce that $\mathrm{u} \rightarrow \mathrm{J}\left(\mathrm{y}_{\mathrm{u}}^{\varepsilon}, \mathrm{u}\right)$ is continuous $(\mathrm{E}, \mathrm{d}) \rightarrow \mathbb{R}$. 
We are now in position to apply Ekeland's Principle. Using spike perturbations (i.e. a perturbed control $v^{\varepsilon}$ equal to $u$ a.e. except on a ball of radius $\varepsilon$ around a given $x^{0} \in \Omega$ ) and applying Proposition 4.3 of [4] we deduce the result.

\section{V - RETURNING TO THE ORIGINAL PROBLEM}

Let $\bar{u}$ be a solution of the original problem (2.3). Put $\bar{y}=y_{\bar{u}}$.

Theorem 5.1 We assume that $\mathrm{K}$ is bounded. Let $\left\{\mathrm{u}^{\varepsilon}\right\}$ be given by Theorem 4.3 applied to $\overline{\mathrm{u}}$ (solution of (2.3)). Then when $\varepsilon>0$ one has

$$
\begin{aligned}
& \mathrm{d}\left(\mathrm{u}^{\varepsilon}, \overline{\mathrm{u}}\right)>0 \\
& \mathrm{y}^{\varepsilon} \rightarrow \overline{\mathrm{y}} \text { in } \mathrm{Y} \text { weak, and } \mathrm{L}^{\infty}(\Omega) \text { strong, }
\end{aligned}
$$

and their exists $\bar{p}$, limit-point in $H_{0}^{1}(\Omega)$ weak and $L^{\infty}(\Omega)$ weak star of $\left\{p^{\varepsilon}\right\}$ and $\rho$, limit-point in $H^{-1}(\Omega)$ weak of $\beta_{\varepsilon}^{\prime}\left(y^{\varepsilon}\right) p^{\varepsilon}$ such that

(5.3) $\left\{\begin{array}{l}A^{*} \bar{p}+\varphi_{y}^{\prime}(., \bar{y}, u) \bar{p}+\rho=L_{y}^{\prime}(., \bar{y}, \bar{u}) \text { in } \Omega \\ \bar{p}=0 \text { on } \Gamma\end{array}\right.$

and for all $v \in K$

$$
H(., \bar{y}, \bar{u}, \bar{p}) \leqslant H(., \bar{y}, v, \bar{p}) \text { a.e. on } \Omega \text {. }
$$

Proof We have, by Theorem 4.3, d( $\left.\mathrm{u}^{\varepsilon}, \overline{\mathrm{u}}\right) \leqslant(C \varepsilon)^{1 / 2} \rightarrow 0$; this proves (5.1). By Theorem 2.1, we deduce that $\left\{y^{\varepsilon}\right\}$ is bounded, hence has a weak limit point $y$, in $Y$, hence $y^{\varepsilon^{k}} \rightarrow y$ in $L^{\infty}(\Omega)$ for a sequence $\varepsilon^{k} \rightarrow 0$. Let $y_{u^{\varepsilon}}$ be the solution of the original state equation associated to $u^{\varepsilon}$. Then as $\mathrm{u} \rightarrow \mathrm{y}_{\mathrm{u}}$ is continuous $:(\mathrm{E}, \mathrm{d}) \rightarrow \mathrm{L}^{\infty}(\Omega)$ (see the proof of Theorem 4.3), we have using Theorem $3.1:$

$$
\|\bar{y}-y\|_{\infty} \leqslant\left\|\bar{y}-y_{u^{\varepsilon}}\right\|_{\infty}+\left\|y_{u^{\varepsilon}}-y_{\varepsilon}\right\|_{\infty} \rightarrow 0
$$

hence $\bar{y}=y$. This proves $(5.2)$.

The boundedness of $\left\{y^{\varepsilon}\right\}$ and $\left\{u^{\varepsilon}\right\}$ in $L^{\infty}$ and (2.9) imply that $L_{y}^{\prime}\left(., y^{\varepsilon}, u^{\varepsilon}\right)$ is bounded in $L^{s}(\Omega)$. This and Lemma 3.2 of [4] imply that $\left\{\mathrm{p}^{\varepsilon}\right\}$ is bounded in $\mathrm{H}_{0}^{1}(\Omega) \cap \mathrm{L}^{\infty}(\Omega)$; Then $\beta_{\varepsilon}^{\prime}\left(\mathrm{y}^{\varepsilon}\right) \mathrm{p}^{\varepsilon}=$ $L_{y}^{\prime}\left(., y^{\varepsilon}, u^{\varepsilon}\right)-\varphi_{y}^{\prime}\left(., y^{\varepsilon}, u^{\varepsilon}\right) p^{\varepsilon}-A p^{\varepsilon}$ is bounded in $L^{s}(\Omega)+H^{-1}(\Omega)=H^{-1}(\Omega)$ (as $s \geqslant 2$ ). 
Let $(\bar{p}, \rho)$ be a limit point of $\left\{\left(p^{\varepsilon}, \beta_{\varepsilon}^{\prime}\left(y^{\varepsilon}\right) p^{\varepsilon}\right)\right\}$ in $H_{0}^{1}(\Omega)$ (weak) $\cap L^{\infty}(\Omega)$ (weak) $\times H^{-1}(\Omega)$ weak. Using (2.7), (2.9) and Lebesgue's Theorem we obtain (5.3).

Now $\mathrm{H}_{0}^{1}(\Omega)$ is compactly embedded in $\mathrm{L}^{2}(\Omega)$; hence $\mathrm{p}^{\varepsilon^{\mathrm{k}}} \rightarrow \overline{\mathrm{p}}$ a.e. on $\Omega$. Using $(2.7)$, (2.9) we deduce that for any sequence $\varepsilon^{k} \rightarrow 0$

$$
\begin{aligned}
& H\left(., y^{\varepsilon^{k}}, u^{\varepsilon^{k}}, p^{\varepsilon^{k}}\right) \rightarrow H(., \bar{y}, \bar{u}, \bar{p}) \\
& H\left(., y^{\varepsilon^{k}}, v, p^{\varepsilon^{k}}\right) \rightarrow H(., \bar{y}, v, \bar{p})
\end{aligned}
$$

As $\alpha^{\varepsilon} \backslash 0$ this allows to pass to the limit in (4.3). This proves (5.4).

We give now some illustrations of our result, considering particular cases for $\beta$. Here we shall get some more properties comparable to those found in D. Tiba [16-18].

First let us assume that $\beta$ is the maximal monotone extension of a monotone simple function defined on some real interval. That is, the graph of $\beta$ is composed only of segments parallel to the axes. We denote

$$
\begin{aligned}
& D=\{r \in R ; r \text { is a discontinuity point of } \beta\} \\
& \Omega_{0}=\{x \in \Omega ; \bar{y}(x) \notin D\}
\end{aligned}
$$

which is an open subset in $\Omega$ since $\bar{y}$ is continuous.

Corollary 5.2 Under the above assumptions, the distribution $\rho$ satisfies supp $\rho \subseteq \Omega \backslash \Omega_{0}$.

Proof We take $\psi \in \mathscr{D}(\Omega)$ arbitrary with supp $\psi \subset \Omega_{0}$ such that

$$
\inf _{x \in \operatorname{supp} \psi} \operatorname{dist}(\bar{y}(x), D) \geqslant 2 c>0 .
$$

It follows that

$$
\inf _{x \in \operatorname{supp} \psi} \operatorname{dist}\left(y^{\varepsilon}(x), D\right) \geqslant c>0
$$

by the uniform convergence of $y^{\varepsilon}$ and for $\varepsilon$ sufficiently small. 
Condition (3.1i) shows that $\beta^{\varepsilon}(r)$ is constant when dist $(r, D) \geqslant c$ and $\varepsilon$ is small. Then $\beta^{\prime} \varepsilon\left(y^{\varepsilon}(x)\right)$ $=0$ for $\mathrm{x} \in \operatorname{supp} \psi$ and $\varepsilon$ sufficiently small. It yields that

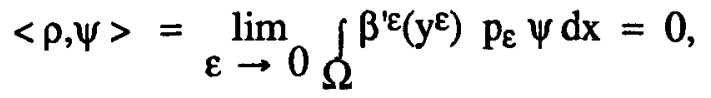

which finishes the proof.

Remark 5.1 From [1] and our hypothesis it follows that the restriction of $\mathrm{p}$ to $\Omega_{0}$ is in $\mathrm{W}_{\mathrm{loc}}^{2, \mathrm{~s}}\left(\Omega_{0}\right)$. If the Hamiltonian is a smooth and uniformly convex function of the control this may give some smoothness results on the control itself. For instance if $K$ is a bounded interval of $\mathbb{R}$ and

$$
\begin{aligned}
& \varphi(\mathrm{x}, \mathrm{y}, \mathrm{u})=\varphi_{1}(\mathrm{x}, \mathrm{y})+\mathrm{u} \\
& \mathrm{L}(., \mathrm{y}, \mathrm{u})=\mathrm{L}_{1}(., \mathrm{y})+(\mathrm{u})^{2}
\end{aligned}
$$

then Pontryagin's principle can be restated as

$$
\bar{u}(x)=-\operatorname{Proj}_{K}(\bar{p}(x)) \text { a.e. on } \Omega
$$

where $\operatorname{Proj}_{K}$ denotes the projection onto K. In this case the restriction of $\bar{u}$ to $\Omega$ is in $W_{l o c}^{1, s}\left(\Omega_{0}\right)$ and in $W_{\text {loc }}^{2, s}$ in regions of $\Omega_{0}$ where $\bar{p}(x)$ is in $\stackrel{\circ}{K}$.

We now consider a generalization of the obstacle problem corresponding to $\beta$ given by

$$
\beta(\Omega)= \begin{cases}0 & r>0 \\ ]-\infty, 0] & r=0 \\ \varnothing & r<0\end{cases}
$$

In this special case we are able to refine the result of Theorem 5.1.

Theorem 5.2 We assume that $\mathrm{K}$ is bounded and that (5.5) holds. Then the conclusion of Theorem 5.1 holds with $\operatorname{supp}(\rho) \subset\{x \in \Omega ; \bar{y}(x)=0\}$ and

$$
\int_{\Omega}[\mathrm{A} \overline{\mathrm{y}}+\varphi(., \overline{\mathrm{y}}, \overline{\mathrm{u}})] \overline{\mathrm{p}} \mathrm{dx}=0 . \square
$$

Proof We can construct $\beta_{\varepsilon}$ as in case 3 of part 3.2 i.e.

$$
\beta_{\varepsilon}(s)= \begin{cases}\varnothing & \text { if } s \leqslant-\varepsilon, \\ \operatorname{tg} \frac{\pi}{2 \vec{\varepsilon}} & \text { if } s \in(-\varepsilon, 0), \\ 0 & \text { if } s \geqslant 0 .\end{cases}
$$


In this case we have

$$
\beta_{\varepsilon}^{\prime}(s)=\frac{\pi}{2 \varepsilon}\left[1+\beta_{\varepsilon}(s)^{2}\right] \geqslant \frac{\pi}{2 \varepsilon} \beta_{\varepsilon}(s)^{2} \text { on dom }\left(\beta_{\varepsilon}\right)
$$

Multiplying (4.2) by $p_{\varepsilon}$ and integrating over $\Omega$ we deduce that $\sqrt{\beta_{\varepsilon}^{\prime}\left(y^{\varepsilon}\right)} p^{\varepsilon}$ is bounded in $L^{2}(\Omega)$, hence with (5.7) that $\varepsilon^{-1 / 2} \beta_{\varepsilon}\left(y^{\varepsilon}\right) p^{\varepsilon}$ is bounded in $L^{2}(\Omega)$ and in particular

$$
\beta_{\varepsilon}\left(y^{\varepsilon}\right) p^{\varepsilon} \rightarrow 0 \text { in } L^{2}(\Omega)
$$

As $\mathrm{H}_{0}^{1}(\Omega)$ is compactly embedded in $\mathrm{L}^{2}(\Omega), \mathrm{p}^{\varepsilon} \rightarrow \overline{\mathrm{p}}$ in $\mathrm{L}^{2}(\Omega)$. On the other hand $\beta_{\varepsilon}\left(y^{\varepsilon}\right)=A y^{\varepsilon}-\varphi\left(., y^{\varepsilon}, u^{\varepsilon}\right)$ is bounded in $L^{s}(\Omega)$. As $y^{\varepsilon} \rightarrow \bar{y}$ in $Y, \varphi\left(., y^{\varepsilon}, u^{\varepsilon}\right) \rightarrow \varphi(., \bar{y}, \bar{u})$ in $L s(\Omega)$ and as $\beta(\bar{y})=A \bar{y}-\varphi(., \bar{y}, \bar{u})$, we have $\beta^{\varepsilon}\left(y^{\varepsilon}\right) \rightarrow \beta(\bar{y})$ in $L s(\Omega)$. Hence as $s \geqslant 2$ $\oint^{\beta^{\varepsilon}\left(y^{\varepsilon}\right) p^{\varepsilon} d x} \rightarrow \oint^{\beta(\bar{y})} \bar{p} d x$. This with (5.8) amounts to (5.6).

Remark 5.3 This result can be compared with the one of Barbu [2], p. 83 in which $\varphi$ is not present.

\section{REFERENCES}

[1] S. Agmon, A. Douglis, L. Nirenberg, Estimates near the boundary for solutions of elliptic partial differential equations satisfying general boundary conditions. Comm. Pure Appl. Math. 12(1959), 623-727.

[2] V. Barbu, Optimal control of variational inequalities, Lecture Notes in Math. 100, Pitman, London 1984.

[3] V. Barbu, D. Tiba, Optimal control of abstract variational inequalities, Proc. Fifth. IFAC Symp. Control of Distributed Parameter Systems, June 26-29, Perpignan A. El Jai ed. (1989).

[4] J.F. Bonnans, E. Casas, Un principe de Pontryagine pour le contrôle des systèmes semilinéaires elliptiques. J. Diff. Equ. (accepted subject to minor corrections).

[5] J.F. Bonnans, E. Casas, Maximum principles in the optimal control of semilinear elliptic systems, Proc. Fifth. IFAC Symp. Control of Distributed Parameter Systems, June 26-29, Perpignan A. El Jai ed. (1989). 
[6] J.F. Bonnans, E. Casas, Optimal control of state constrained multistate systems, SIAM J. Control Optimiz. 27(1989), 446-455.

[7] H. Brezis, Problèmes unilatéraux, J.Math. Pures Appl. 51(1972), 1-168.

[8] F.H. Clarke, Optimization and nonsmooth analysis, J. Wiley, New York, 1983.

[9] I. Ekeland, Sur les problèmes variationnels, C.R. Acad. Sci. Paris 275, (1972), 1057-1059.

[10] I. Ekeland, Nonconvex minimization problems, Bull. Amer. Math. Soc. 1 (NS) (1979), 447 474.

[11] A. Haraux, How to differentiate the projection on a convex set in Hilbert space. Some applications to variational inequalities. J. Math. Soc. Japan 29 (1977), 615-631.

[12] A.D. Ioffe, V. Tihomirov, Theory of extremal problems, Nauka, Moscou, 1974 (in Russian ; English translation :North Holland, 1979).

[13] D. Kinderlehrer, G. Stampacchia (1980). An introduction to variational inequalities and their applications. Academic Press, New York.

[14] F. Mignot, Contrôle dans les inéquations variationnelles elliptiques. J. Funct. Anal. 22 (1976), 130-185.

[15] C. Saguez, Contrôle optimal de systèmes à frontière libre. Thèse d'état, Université de Compiègne, 1980.

[16] D. Tiba, Quelques remarques sur le contrôle de la corde vibrante avec obstacle. C.R.Acad. Sci. Paris, 229-1, 13 (1984).

[17] D. Tiba, Some remarks on the control of the vibrating string with obstacle. Rev. Roum. Math. Pures et Appl. 29, 10 (1984), 899-906.

[18] D. Tiba, Optimal control for second order semilinear hyperbolic equations, Control-Theory and Advanced Technology, 3 (1987), $33-43$,.

[19] J.P. Yvon, Etude de quelques problèmes de contrôle pour des systèmes distribués. Thèse d'état, Université de Paris VI, 1973. 


\section{APPENDIX}

The following result is stated, but without proof, in Brezis [7, p. 17]. Hence, although it is well known by the specialists of the field we find convenient to give a proof of it.

Theorem A.1 The equation

$$
\left\{\begin{array}{l}
A y+\beta(y(x)) \\
y=0 \text { on } \Gamma
\end{array}\right.
$$

where $\mathrm{A}$ is a differential operator satisfying (2.4)-(2.5), $\boldsymbol{\beta}$ is a maximal monotone graph in $\mathbf{R} \times \mathbf{R}$ with $\beta(0) \ni 0$ and $f \in L^{s}(\Omega)$ with $s \geqslant 2$, has a unique solution $y \in W^{2}, s(\Omega) \cap H_{0}^{1}(\Omega)$ and there exists $C>0$ depending only on $\Omega$ and $A$ (not on $\beta$ and $f$ ) such that $\|y\|_{W} 2, s(\Omega) \leqslant C\|f\|_{L s}(\Omega)$.

\section{Proof}

a) A priori estimate. Let us assume that $\beta$ is Lipschitz. Define for $\sigma \in R$, $F(\sigma):=|\beta(\sigma)|^{s-2} \beta(\sigma)$. Then $F(\sigma)$ is Lipschitz and $F^{\prime}(\sigma)=(s-1)|\beta(\sigma)|^{s-2} \beta^{\prime}(\sigma)$ a.e. on R. Also $\mathrm{F}(\mathrm{y}(\mathrm{x}))$ is in $\mathrm{H}_{0}^{1}(\Omega)$ and its gradient is $\mathrm{F}^{\prime}(\mathrm{y}(\mathrm{x})) \nabla \mathrm{y}(\mathrm{x})$ a.e. on $\Omega$. Multiplying by $\mathrm{F}(\mathrm{y}(\mathrm{x}))$ the equation and integrating by parts we obtain using the fact that $\beta(0) \ni 0$ :

$$
\begin{aligned}
(s-1) & \left\{\left.|\beta(y(x))|\right|^{s-2} \beta^{\prime}(y(x)) \sum_{i, j=1}^{n} a_{i j}(x) \frac{\partial y(x)}{\partial x_{i}} \frac{\partial y(x)}{\partial x_{j}} d x+\oint_{\Omega}|\beta(y(x))|^{s} d x\right. \\
& =\oint^{f}|\beta(y(x))|^{s-2} \beta(y(x)) d x .
\end{aligned}
$$

From the positivity of the first term and Hölder's inequality we get

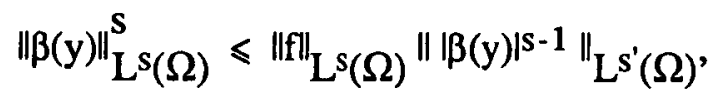

with $1 / \mathrm{s}+1 / \mathrm{s}^{\prime}=1$. But using $s=(\mathrm{s}-1) \mathrm{s}^{\prime}=1+\mathrm{s} / \mathrm{s}^{\prime}$ we get

$$
\left\||\beta(y)|^{s-1}\right\|_{L^{s^{\prime}}(\Omega)}=\left(\int_{\Omega} \mid \beta\left(\left.y(x)\right|^{s} d x\right)^{1 / s^{\prime}}=\|\beta(y)\|_{L^{s}(\Omega)}^{s / s^{\prime}}=\|\beta(y)\|_{L}^{s-1}(\Omega)\right.
$$


This with (A.1) imply $\|\beta(y)\|_{L s(\Omega)} \leqslant\|f\|_{L s(\Omega)}$, which in turn this implies $\|A y\|_{L} s(\Omega) \leqslant 2\|f\|_{L s(\Omega)}$. Using the classical results of Agmon et al. [1] we get the desired apriori estimate.

b) Construction of the solution. To $\beta$ is associated its Yosida approximate $\beta_{\varepsilon}$ (here $\varepsilon>0$ is a small parameter (see [2]). Now $\beta_{\varepsilon}$ has Lipschitz constant $1 / \varepsilon$ and $\beta_{\varepsilon}(0)=0$. The perturbed equation

$$
\left\{\begin{array}{l}
A y_{\varepsilon}+\beta_{\varepsilon}\left(y_{\varepsilon}(x)\right) \ni f(x) \text { in } \Omega \\
y_{\varepsilon}=0 \text { on } \Gamma
\end{array}\right.
$$

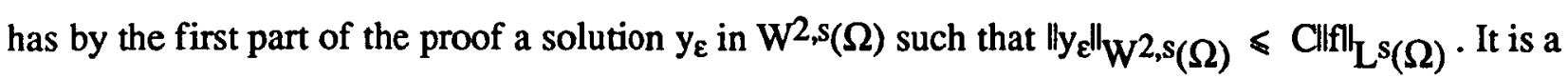
standard process to pass to the limit in the state equation when $\varepsilon>0$ : let y be a weak limit-point in $\mathrm{W}^{2}, \mathrm{~s}(\Omega)$ of $\left\{\mathrm{y}_{\varepsilon}\right\}$, then $\|y\|_{\mathrm{W}^{2}, \mathrm{~s}(\Omega)} \leqslant \mathrm{C}\|f\|_{\mathrm{L}(\Omega)}$ and $\mathrm{y}$ is a solution of the state equation. The unicity of the solution is a consequence of the strict monotonicity of the operator. 
